\title{
Can a multivalent defect be mimicked by several Shockley-Read-Hall-like defects?
}

\author{
Koen Decock, ${ }^{\text {a) }}$ Samira Khelifi, and Marc Burgelman \\ Department of Electronics and Information Systems (ELIS), Universiteit Gent, St-Pietersnieuwstraat 41, \\ B-9000 Gent, Belgium
}

(Received 14 June 2010; accepted 6 August 2010; published online 21 September 2010)

\begin{abstract}
The origin of defects present in a device is not always clear. Hence, in electronic device simulation, the influence of defects with more than two different charge states (multivalent defects) is often modeled as a set of defects with only two possible charge states [Shockley-Read-Hall (SRH)-like defects] which follow the SRH statistics. This paper investigates under which circumstances this procedure is allowed, and provides means to check the equivalence between the multivalent and SRH-like description in a fast and efficient way. The procedures are verified simulating a thin film solar cell structure. (ㅇ 2010 American Institute of Physics. [doi:10.1063/1.3487474]
\end{abstract}

\section{INTRODUCTION}

Multivalent defects, i.e., defects with more than two possible charge states, ${ }^{1}$ are important in several material systems used in solar cell production. ${ }^{2-5}$ The statistics governing this kind of defects differs from the usual Shockley-Read-Hall statistics (SRH) for defects with only two possible charge states, ${ }^{1}$ which can lead to a different recombination rate. ${ }^{6}$ The main difference between recombination through a multivalent and a SRH-like defect is the fact that the number of recombination centers linked with the transition between two charge states is fixed in the SRH case, whereas it is dependent on the free carrier densities (and thus on the position of the Fermi levels) in the multivalent case. This dependence can lead to the disappearance of some recombination paths which would not disappear if they were part of a set of SRHlike defects. When the electron $(n)$ and hole $(p)$ density is known however, the recombination can be described as proceeding via several sets of SRH-recombination centers. Because the appropriate defect densities of these corresponding sets vary with the local electron and hole concentration it is impossible to a priori mimic the recombination of a multivalent defect with SRH-like defects but often a good correspondence can be found setting the defect densities of each SRH-like defect the same as the total defect density of the multivalent defect.

This paper investigates under which circumstances a multivalent defect can be modeled as a set of SRH-like defects. First the recombination rate diagrams of a multivalent defect and of a set of SRH-like defects are discussed and compared. Next the relation between the recombination rate for a multivalent defect with three charge states and the recombination rate for its SRH equivalent is investigated. The conditions under which such a defect can be mimicked by a set of SRH-like defects are stated afterwards. Finally the results are verified using SCAPS 2.9, a numerical solar cell simulation tool of the University of Gent available to the photovoltaic research community.

${ }^{a)}$ Electronic mail: koen.decock@elis.ugent.be.

\section{DEFINITIONS AND ASSUMPTIONS}

Partially following the notation of Sah and Shockley, ${ }^{1}$ the different charge states are designated with a subscript $s$ representing the number of negative charges on the defect. The different transitions are designated with a superscript which is the mean value of the charge states involved, e.g., $s+1 / 2$ represents the transitions (in both directions) between the charge states $s$ and $s+1$. Whenever the distinction should be made between the multivalent and SRH-like nature of the defect statistics used, the former will be designated here with a subscript MUL And the latter with a subscript SRH.

Instead of using the emission constants $e_{n}$ and $e_{p}$, the fictitious charge densities $n^{*}$ and $p^{*}$ (Ref. 1) will be used (1).

$$
\begin{aligned}
& n^{*, s+1 / 2}=\frac{e_{p}^{s+1 / 2}}{c_{n}^{s+1 / 2}}, \\
& p^{*, s+1 / 2}=\frac{e_{n}^{s+1 / 2}}{c_{p}^{s+1 / 2}} .
\end{aligned}
$$

With $c_{n}$ and $c_{p}$ the capture constants.

The emission constants are assumed to be equal in the multivalent and SRH-like case. This implies that the energy levels of the SRH-like defects should be slightly shifted with respect to the corresponding multivalent level, in order to account for possible degeneracy factors. ${ }^{8,9}$

The defect density is assumed to be sufficiently low with respect to the shallow doping density, in order to ensure that its influence on the free carrier concentrations is negligible. As will be discussed at the end of the paper, this assumption is not stringent.

Nonequilibrium but steady-state conditions are assumed. The net steady-state recombination rate linked with transitions between the charge levels $s$ and $s+1$ is noted as $U^{s+1 / 2}$.

Following the notation used by Sah and Shockley, ${ }^{1}$ the ratio between the occupation probability of two successive charge levels will be designated as $R_{N}$, and $N_{s}$ represents the density of a defect in charge state $s$. Sah and Shockley have proven that (2) holds for both SRH-like and multivalent defects. 


$$
R_{N}^{s+1 / 2}=\frac{N_{s+1}}{N_{s}}=\frac{c_{n}^{s+1 / 2}}{c_{p}^{s+1 / 2}} \frac{n+n^{*, s+1 / 2}}{p+p^{*, s+1 / 2}} .
$$

\section{AN EQUIVALENT SET OF SRH-LIKE DEFECTS}

The recombination rates for one of the energy levels of a multivalent defect and for a SRH-like defect are shown in (3), with $N_{t}$ the total defect density.

$$
\begin{aligned}
U_{\mathrm{MUL}}^{s+1 / 2}= & \frac{n p-n_{i}^{2}}{\left(p+p^{*, s+1 / 2}\right) / c_{n}^{s+1 / 2}+\left(n+n^{*, s+1 / 2}\right) / c_{p}^{s+1 / 2}} \\
& \times\left(N_{s}+N_{s+1}\right), \\
U_{\mathrm{SRH}}^{s+1 / 2}= & \frac{n p-n_{i}^{2}}{\left(p+p^{*, s+1 / 2}\right) / c_{n}^{s+1 / 2}+\left(n+n^{*, s+1 / 2}\right) / c_{p}^{s+1 / 2}} N_{t} .
\end{aligned}
$$

For each level at each position in a semiconductor layer the multivalent recombination rate can be mimicked by a set of SRH-like defects if one chooses $N_{t}=N_{s}+N_{s+1}$. As one does not a priori know the occupation probabilities of the different charge states, this approach is impossible to be followed in practice. The best possible SRH-like equivalent of a multivalent defect is to use a set of defects having the same capture and emission constants as the different levels of the multivalent defect and each SRH-like defect having the same defect density as the entire multivalent defect. The recombination rate for levels which are linked with a charge level which is almost entirely occupied is then correctly modeled. The recombination rate for levels linked with charge levels which are both almost entirely unoccupied is then overestimated. The consequences of this deviation are discussed in this paper.

\section{THE RECOMBINATION RATE DIAGRAMS, $\boldsymbol{R}_{U}$-DIAGRAMS}

In order to evaluate the relative importance of the net steady-state recombination rate of two adjacent energy levels Sah and Shockley ${ }^{1}$ defined the ratio between both as $R_{U}$ and represented it in an $R_{U}$-diagram. This paper presents a fast and easy way to construct these diagrams. Additionally, an analogous diagram for a set of two SRH-like defects is developed, leading to different but similar results.

The construction of the $R_{U}$-diagrams for the multivalent defect and its SRH-like equivalent allows a fast inspection of the (non)equivalence between both approaches.

\section{A. Multivalent}

The ratio of the net recombination rate of two adjacent energy levels for a multivalent defect $R_{U}$ has been calculated by Sah and Shockley ${ }^{1}$ as (4).

$$
R_{U, \mathrm{MUL}}=\frac{U^{s+1 / 2}}{U^{s-1 / 2}}=\frac{c_{n}^{s+1 / 2}}{c_{p}^{s-1 / 2}} \frac{n+n^{*, s-1 / 2}}{p+p^{*, s+1 / 2}} .
$$

In order to visualize this expression Sah and Shockley drew

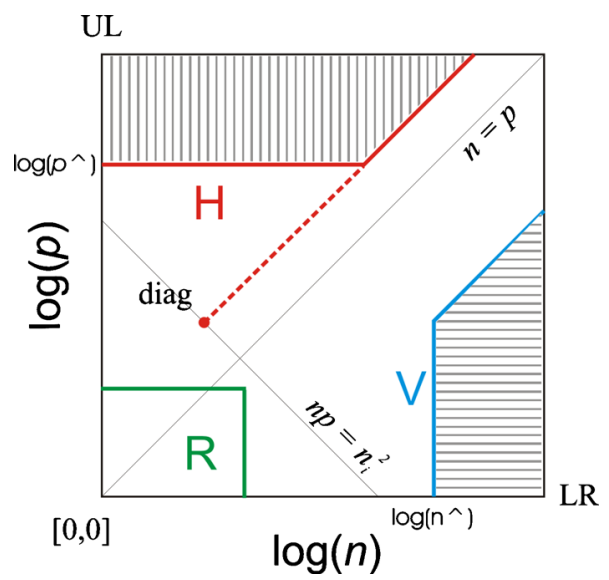

FIG. 1. (Color online) $R_{U}$-diagram with the possible curve types of the $R_{U}=1$-line. For $R_{U, \mathrm{MUL}}$ two curve types (H-type and V-type) are possible, for $R_{U, \mathrm{SRH}}$ a third type (R-type) is possible. The line corresponding with thermal equilibrium $\left(n p=n_{i}^{2}\right)$ and charge equality $(n=p)$ are indicated. For the H-type curve the intersection of the extension of the diagonal part of the curve with the line corresponding with thermal equilibrium is marked (diag). The meaning of "above" and "below" is illustrated with dashed regions. The region above the H-type curve is horizontally dashed, the region below the V-type curve is vertically dashed.

the line corresponding to $R_{U}=1$ in a diagram with the logarithm of the electron density on the abscissa and the logarithm of the hole density on the ordinate. This paper expounds on the main characteristics of this curve and provides means for an easy construction.

Two possible shapes for this curve are possible, it can be either diagonal for high electron concentrations and horizontal for low electron concentrations (designated as H-type) or diagonal for high hole concentrations and vertical for low hole concentrations (designated as V-type), both possibilities are shown in Fig. 1. For $[n, p]$-combinations which lie above this line (see, e.g., the vertically dashed region in Fig. 1 for H-type) the recombination is governed by the $s-1 / 2$ level $\left(R_{U}<1\right)$, for $[n, p]$-combinations below this line (see, e.g., the horizontally dashed region in Fig. 1 for V-type) the recombination is governed by the $s+1 / 2$ level $\left(R_{U}>1\right)$. In order to determine whether the curve is of the H-type or V-type it suffices to calculate $R_{U}$ for very small $n$ and $p$, values, as shown in (5) with $E_{i}$ the intrinsic energy level. If $R_{U}^{[0,0]}<1$ the curve is $\mathrm{V}$-type, otherwise it is H-type.

$$
R_{U}^{[0,0]}=\frac{c_{n}^{s+1 / 2} n^{*, s-1 / 2}}{c_{p}^{s-1 / 2} p^{*, s+1 / 2}}=\frac{c_{p}^{s+1 / 2}}{c_{p}^{s-1 / 2}} \exp \left(\frac{2 E_{i}-E^{s+1 / 2}-E^{s-1 / 2}}{k T}\right) .
$$

For both H-type and V-type, the curve has a kink at high free carrier densities, and becomes a line of slope +1 in the double logarithmic diagram. The exact position of this diagonal part is determined by the intersection of (the extrapolation of) this curve and the line representing thermal equilibrium. This intersection point is shown in (6) and indicated in Fig. 1. 


$$
[n, p]_{\mathrm{diag}}=\left[n_{i} \sqrt{\frac{c_{p}^{s-1 / 2}}{c_{n}^{s+1 / 2}}}, n_{i} \sqrt{\frac{c_{n}^{s+1 / 2}}{c_{p}^{s-1 / 2}}}\right]
$$

The $n$-value of the horizontal part of the curve $\left(n^{\wedge}\right)$ for the H-type case or the $p$-value of the vertical part of the curve $\left(p^{\wedge}\right)$ for the V-type case are shown in (7).

$$
\begin{aligned}
& n^{\wedge}=\frac{c_{p}^{s-1 / 2} p^{*, s+1 / 2}-c_{n}^{s+1 / 2} n^{*, s-1 / 2}}{c_{n}^{s+1 / 2}}, \\
& p^{\wedge}=\frac{c_{n}^{s+1 / 2} n^{*, s-1 / 2}-c_{p}^{s-1 / 2} p^{*, s+1 / 2}}{c_{p}^{s-1 / 2}} .
\end{aligned}
$$

Calculating the abovementioned values, the $R_{U}$-diagram can be drawn in a fast and easy way.

\section{B. SRH-like}

Following a similar procedure as Sah and Shockley did for two levels of a multivalent defect, the expression of $R_{U}$ for two separate SRH-like defects with an equal defect density is calculated in (8).

$$
\begin{aligned}
R_{U, \mathrm{SRH}} & =\frac{U^{s+1 / 2}}{U^{s-1 / 2}} \\
& =\frac{c_{n}^{s+1 / 2} c_{p}^{s+1 / 2}}{c_{n}^{s-1 / 2} c_{p}^{s-1 / 2}} \frac{c_{n}^{s-1 / 2}\left(n+n^{*, s-1 / 2}\right)+c_{p}^{s-1 / 2}\left(p+p^{*, s-1 / 2}\right)}{c_{n}^{s+1 / 2}\left(n+n^{*, s+1 / 2}\right)+c_{p}^{s+1 / 2}\left(p+p^{*, s+1 / 2}\right)} .
\end{aligned}
$$

Plotting the $R_{U}=1$-line, leads to similar results as for $R_{U \text {,MUL }}$. However, next to H-type and V-type, a third possible shape appears (R-type), also shown in Fig. 1. In order to determine the shape of the curve one should first calculate $R_{U}$ in the upper left (UL) and lower right (LR) corner of the graph, corresponding with a $p$-dominated or $n$-dominated situation, respectively. These expressions are shown in (9).

$$
R_{U}^{\mathrm{UL}}=\frac{c_{n}^{s+1 / 2}}{c_{n}^{s-1 / 2}} ; R_{U}^{\mathrm{LR}}=\frac{c_{p}^{s+1 / 2}}{c_{p}^{s-1 / 2}} .
$$

One also needs to calculate $R_{U}$ in depletion, shown in (10), where $n^{*} \gg n$ and $p^{*} \gg p$, are assumed in depletion.

$$
\begin{aligned}
R_{U}^{[0,0]}= & \frac{c_{n}^{s+1 / 2} c_{p}^{s+1 / 2}}{c_{n}^{s-1 / 2} c_{p}^{s-1 / 2}} \frac{c_{n}^{s-1 / 2} n^{*, s-1 / 2}+c_{p}^{s-1 / 2} p^{*, s-1 / 2}}{c_{n}^{*+1 / 2} n^{*+1 / 2}+c_{p}^{s+1 / 2} p^{*, s+1 / 2}} \\
= & \frac{c_{p}^{s+1 / 2}}{c_{p}^{s-1 / 2}} \frac{1+c_{p}^{s-1 / 2} / c_{n}^{s-1 / 2} \exp \left(\left(2 E_{i}-2 E^{s-1 / 2}\right) / k T\right)}{1+c_{p}^{s+1 / 2} / c_{n}^{s+1 / 2} \exp \left(\left(2 E_{i}-2 E^{s+1 / 2}\right) / k T\right)} \\
& \times \exp \left(\frac{E^{s-1 / 2}-E^{s+1 / 2}}{k T}\right) .
\end{aligned}
$$

If the signs of the logarithms of $R_{U}^{\mathrm{UL}}, R_{U}^{\mathrm{LR}}$, and $R_{U}^{[0,0]}$ are equal, the $R_{U}=1$-curve does not exist and one of both defects dominates the other in the entire $[n, p]$-space. If the signs of the logarithms of $R_{U}^{\mathrm{UL}}$ and $R_{U}^{\mathrm{LR}}$ are the same but opposite to the one of $R_{U}^{[0,0]}$, the shape of the curve is R-type. If the signs of the logarithms of $R_{U}^{\mathrm{UL}}$ and $R_{U}^{\mathrm{LR}}$ are opposite, the curve is $\mathrm{H}$-type or V-type. In order to discriminate between H-type and V-type one should compare the signs of the logarithms of $R_{U}^{[0,0]}$ and $R_{U}^{\mathrm{LR}}$, if they are the same the curve is H-type, otherwise it is V-type. The logarithm of $R_{U}$ below the curve ("below" having the same meaning as in the multivalent case) has the same sign as the logarithm of $R_{U}^{\mathrm{LR}}$. Hence if $R_{U}^{\mathrm{LR}}>1$, the logarithm of $R_{U, \mathrm{SRH}}$ has the same sign above/ below the $R_{U}=1$-line as in the multivalent case $\left(R_{U, \mathrm{MUL}}\right)$.

The coordinates of the intersection of (the extension of) the $R_{U}=1$-curve and the line representing the thermal equilibrium are given in (11).

$$
[n, p]_{\mathrm{diag}}=\left[n_{i} \sqrt{\frac{1 / c_{n}^{s-1 / 2}-1 / c_{n}^{s+1 / 2}}{1 / c_{p}^{s+1 / 2}-1 / c_{p}^{s-1 / 2}}}, n_{i} \sqrt{\frac{1 / c_{p}^{s+1 / 2}-1 / c_{p}^{s-1 / 2}}{1 / c_{n}^{s-1 / 2}-1 / c_{n}^{s+1 / 2}}}\right] .
$$

This expression has only meaning when the curve is H-type or V-type and in these cases it is easily checked that these coordinates are real numbers.

The $n$-value of the horizontal part of the curve or the $p$-value of the vertical part of the curve are calculated in (12).

$$
\begin{aligned}
& n^{\wedge}=\frac{n^{*, s-1 / 2} / c_{p}^{s-1 / 2}+p^{*, s-1 / 2} / c_{n}^{s-1 / 2}-n^{*, s+1 / 2} / c_{p}^{s+1 / 2}-p^{*, s+1 / 2} / c_{n}^{s+1 / 2}}{1 / c_{p}^{s+1 / 2}-1 / c_{p}^{s-1 / 2}}, \\
& p^{\wedge}=\frac{p^{*, s-1 / 2} / c_{n}^{s-1 / 2}+n^{*, s-1 / 2} / c_{p}^{s-1 / 2}-p^{*, s+1 / 2} / c_{n}^{s+1 / 2}-n^{*, s+1 / 2} / c_{p}^{s+1 / 2}}{1 / c_{n}^{s+1 / 2}-1 / c_{n}^{s-1 / 2}} .
\end{aligned}
$$

From the discussion above it is already clear that even though the expressions of the recombination rate for a multivalent defect and its SRH-like equivalent bear a strong resemblance, the relations between the recombination of the different levels can differ significantly. Comparing both $R_{U}$-diagrams gives an indication whether the equivalent set of SRH-like defects is a good representative for the multivalent defect. 


\section{COMPARISON OF THE RECOMBINATION RATES, $\boldsymbol{R}_{W}$-DIAGRAM}

In order to compare whether the recombination rate of the multivalent defect is the same as for the equivalent set of SRH-like defects one should investigate the ratio between both in the entire $[n, p]$-space, noted as $R_{W}(13)$.

$$
R_{W}=\frac{U_{\mathrm{SRH}}}{U_{\mathrm{MUL}}}=\frac{\sum_{s} U_{\mathrm{SRH}}^{s+1 / 2}}{\sum_{s} U_{\mathrm{MUL}}^{s+1 / 2}} .
$$

In this paper a formalism for $R_{W}$ is developed and investigated for a multivalent defect with three possible charge levels $(s-1, s, s+1)$. The defect density is assumed to be suffi- ciently low as to have no influence on the free carrier density. Hence the free carrier concentrations can be assumed to be the same in both the multivalent and SRH-like case, $R_{W}$ can be re-expressed as (14).

$$
R_{W}=\frac{1}{1-N_{s+1} / N_{t}} \frac{1+R_{U, \mathrm{SRH}}}{1+R_{U, \mathrm{MUL}}}=\frac{1}{1-N_{s-1} / N_{t}} \frac{1+1 / R_{U, \mathrm{SRH}}}{1+1 / R_{U, \mathrm{MUL}}},
$$

where $N_{2} / N_{t}$ and $N_{0} / N_{t}$ are easily found as a function of $R_{N}$. It is instructive to rewrite the first factors of Eq. (14) formatted as $(1+a)$ as shown in (15).

$$
\begin{aligned}
& \frac{1}{1-N_{s+1} / N_{t}}=1+\left(\frac{c_{n}^{s+1 / 2}\left(n+n^{*, s+1 / 2}\right)}{c_{p}^{s+1 / 2}\left(p+p^{*, s+1 / 2}\right)} \frac{c_{n}^{s-1 / 2}\left(n+n^{*, s-1 / 2}\right)}{c_{n}^{s-1 / 2}\left(n+n^{*, s-1 / 2}\right)+c_{p}^{s-1 / 2}\left(p+p^{*, s-1 / 2}\right)}\right) \\
& \frac{1}{1-N_{s-1} / N_{t}}=1+\left(\frac{c_{p}^{s-1 / 2}\left(p+p^{*, s-1 / 2}\right)}{c_{n}^{s-1 / 2}\left(n+n^{*, s-1 / 2}\right)} \frac{c_{p}^{s+1 / 2}\left(p+p^{*, s+1 / 2}\right)}{c_{n}^{s+1 / 2}\left(n+n^{*, s+1 / 2}\right)+c_{p}^{s+1 / 2}\left(p+p^{*, s+1 / 2}\right)}\right)
\end{aligned}
$$

It is obvious that the recombination rate due to the equivalent set SRH-like defects always exceeds the recombination rate of the multivalent defect $\left(R_{W}>1\right)$, however as long as $R_{W}$ is close to one the multivalent defect can be modeled as a set of SRH-like defects. In order to see whether a multivalent defect can be mimicked by a set of SRH-like defects one should calculate $R_{W}$ in the entire $[n, p]$-space, or at least in that part of it which is of interest. As $R_{W}>1$, it is impossible to draw the lines corresponding to $R_{W}=1$ but one can draw curves of constant $R_{W}$. Except in some noncommon situations these curves will again be either H-type, V-type, or R-type.

Because the free electron and hole concentration are only present in $R_{W}$ as $\left(n+n^{*}\right)$ and $\left(p+p^{*}\right)$ and because only additions and divisions are present, $R_{W}$ will be much larger than one if and only if it is much larger than one in either the $\mathrm{UL}\left(R_{W}^{\mathrm{UL}}\right)$, the LR $\left(R_{W}^{\mathrm{LR}}\right)$, or the lower left $\left(R_{W}^{[0,0]}\right)$ corner of the $[n, p]$-space. The former two cases are easily calculated as in the UL case one finds $N_{s-1} \approx N_{t}$ and in the LR case one finds $N_{s+1} \approx N_{t}$, the resulting expressions are shown in (16) and (17).

$$
\begin{aligned}
& R_{W}^{\mathrm{UL}}=1+\frac{c_{n}^{s+1 / 2}}{c_{n}^{s-1 / 2}}, \\
& R_{W}^{\mathrm{LR}}=1+\frac{c_{p}^{s-1 / 2}}{c_{p}^{s+1 / 2}} .
\end{aligned}
$$

If $c_{n}^{s+1 / 2} \gg c_{n}^{s-1 / 2}$ the equivalence between the multivalent defect and the set of SRH-like defects will not hold for $p$-dominated situations, if $c_{p}^{s-1 / 2} \gg c_{p}^{s+1 / 2}$ it will not hold for $n$-dominated situations.

The expression for $R_{W}^{[0,0]}$ is more complicated as it is difficult to a priori determine whether $N_{s+1}$ or $N_{s-1}$ is the main charge state. As (14) can be written in the form shown in (18), using (15), one can however state a condition which is easily calculated (19).

$$
\begin{aligned}
& R_{W}=\frac{(1+a)(1+b)}{(1+c)}, \\
& R_{W}^{[0,0]} \gg 1 \Leftrightarrow \max (a, b) \gg \max (c, 1) .
\end{aligned}
$$

In these expressions $(1+a), b$, and $c$ represent either $(1$ $\left.+N_{s+1} / N_{t}\right)^{-1}, \quad R_{U, \mathrm{SRH}}, \quad$ and $R_{U, \mathrm{MUL}}$ or $\left(1+N_{s-1} / N_{t}\right)^{-1}$, $\left(R_{U, \mathrm{SRH}}\right)^{-1}$, and $\left(R_{U, \mathrm{MUL}}\right)^{-1}$, respectively.

\section{COMPARISON WITH NUMERICAL SIMULATION}

As an example and in order to check the applicability of the theory described, a solar cell structure with a multivalent defect in the absorber layer is simulated twice, once taking into account the multivalent nature of the defect and once describing the defect as a set of two SRH-like defects.

\section{A. Numerical details}

The simulations are performed with SCAPS, ${ }^{7}$ version 2.9 is able to model multivalent defects. ${ }^{8}$ The investigated structure represents a $\mathrm{Cu}(\mathrm{In}, \mathrm{Ga})(\mathrm{S}, \mathrm{Se})_{2}$-based solar cell and is based on the model "NUMOS CIGS baseline.def," is distributed together with the installation package of SCAPS. In the absorber a multivalent defect with three possible charge states $(-1 ; 0 ; 1)$ has been introduced, leading to two possible transitions $(-1 / 2 ; 1 / 2)$. The energy levels are at $E^{-1 / 2}=0.45 \mathrm{eV}$ and $E^{1 / 2}=0.65 \mathrm{eV}$ (using the valence band as the reference energy level). The defect density equals $10^{15} \mathrm{~cm}^{-3}$, which is sufficiently smaller than the shallow 


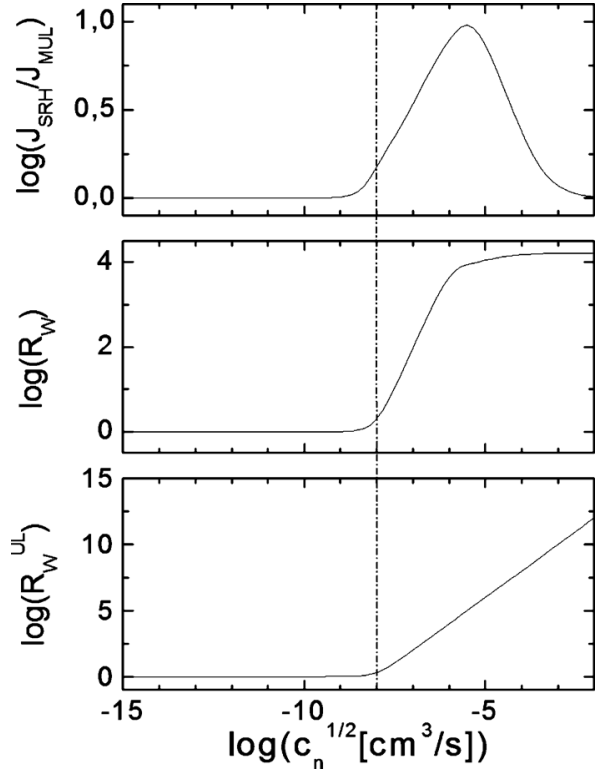

FIG. 2. The effect of the variation in $c_{n}^{1 / 2}=c_{p}^{-1 / 2}$ together with $c_{n}^{-1 / 2}=c_{p}^{1 / 2}$ so that $c_{n}^{-1 / 2} c_{n}^{1 / 2}=10^{-16} \mathrm{~cm}^{6} / \mathrm{s}^{2}$ on the current and on $R_{W}$. For $c_{n}^{1 / 2}$ $<10^{-8} \mathrm{~cm}^{3} / \mathrm{s}$ the set of SRH-like defects is able to represent the multivalent defect. Simulations are performed under dark conditions at $0.25 \mathrm{~V}$ forward bias. In the upper graph the currents are compared. In the middle graph the recombination rates at a certain position in the quasineutral region of the absorber are compared. In the lower graph $R_{W}$ in the UL corner of the $[n, p]$-space is displayed.

doping density $\left(N_{A}=2 \times 10^{16} \mathrm{~cm}^{-3}\right)$. The capture cross sections are varied according to $c_{n}^{-1 / 2}=c_{p}^{1 / 2}, c_{n}^{1 / 2}=c_{p}^{-1 / 2}$, and $c_{n}^{-1 / 2} c_{n}^{1 / 2}=10^{-16} \mathrm{~cm}^{6} / \mathrm{s}^{2}$.

\section{B. Results}

According to the lower graph of Fig. 2 and the theory, the description of the multivalent defect as a set of SRH-like defects is valid as long as $c_{n}^{1 / 2}<10^{-8} \mathrm{~cm}^{3} / \mathrm{s}$. As $c_{n}^{1 / 2}$ becomes larger, both $R_{W}^{\mathrm{UL}}$ and $R_{W}^{\mathrm{LR}}$ (not displayed) significantly exceed one. $R_{W}$ is simulated at a certain point in the quasineutral region of the absorber layer, this is shown in the middle graph of Fig. 2, the point itself is marked on Fig. 3 and is representative for a large part of the absorber. As in the graph of $R_{W}^{\mathrm{UL}}, c_{n}^{1 / 2}=10^{-8} \mathrm{~cm}^{3} / \mathrm{s}$ again is a breaking point. The condition imposed on $R_{W}^{\mathrm{UL}}$ is a good measure for $R_{W}$ in the upper part of the $[n, p]$-space. The upper graph of Fig. 2 shows the relation between the simulated current of the structure with a multivalent defect and the structure with an equivalent set of SRH-like defects. As in the two lower curves of the figure, it can be seen that the equivalence breaks down at $c_{n}^{1 / 2}$ $=10^{-8} \mathrm{~cm}^{3} / \mathrm{s}$. However, for very large capture cross sections $\left(c_{n}^{1 / 2}>10^{-3} \mathrm{~cm}^{3} / \mathrm{s}\right)$ the discrepancy between $J_{\mathrm{SRH}}$ and $J_{\mathrm{MUL}}$ disappears again as the current in the structure then no longer is determined by the bulk recombination rate but rather by the recombination rate at back contact. However, such very large capture cross sections are no longer physical. The most pronounced nonequivalence between $J_{\mathrm{SRH}}$ and $J_{\mathrm{MUL}}$ appears at $c_{n}^{1 / 2}=3 \times 10^{-6} \mathrm{~cm}^{3} / \mathrm{s}$ where the current is overestimated by a factor 10 , and the recombination rate by a factor $10^{4}$.

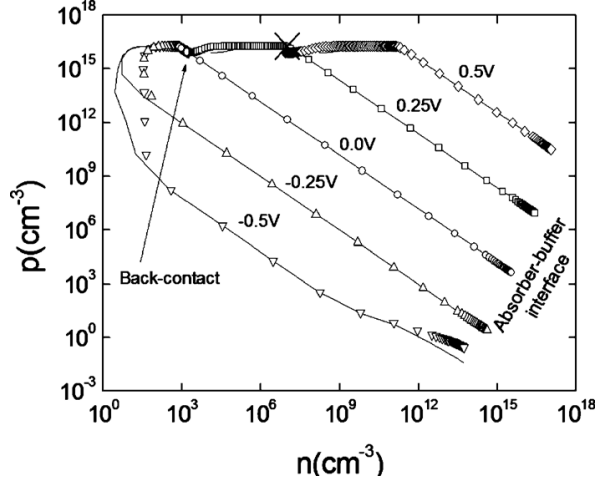

FIG. 3. Paths in the $[n, p]$-space corresponding with the free carrier densities in the absorber with $c_{n}^{1 / 2}=c_{p}^{-1 / 2}=10^{-6} \mathrm{~cm}^{3} / \mathrm{s}^{2}$ and $c_{n}^{-1 / 2}=c_{p}^{1 / 2}$ $=10^{-10} \mathrm{~cm}^{3} / \mathrm{s}$. The simulations with the multivalent defect in solid lines, with the equivalent set of SRH-like defects in symbols. Each symbol represents a point of the mesh used in the simulation, the points corresponding to the absorber-buffer interface and the back contact are indicated. The bias voltage is varied from $0.5 \mathrm{~V}$ (upper right curve) to $-0.5 \mathrm{~V}$ (lower left curve). The point where the recombination was calculated in Fig. 2 is indicated with a cross.

\section{Paths in the $[n, p]$-space}

The $[n, p]$-paths representing the free carrier densities at varying positions in the absorber are displayed in Fig. 3 for different bias voltages. These paths are useful in order to see whether the free carrier density is the same in both the multivalent and the SRH-like case. Moreover they are vital to see which parts of the $[n, p]$-space are of importance in the simulated structure.

All curves exhibit an oblique side corresponding to (20),

$$
n p=n_{i}^{2} \exp \left(\frac{q V}{k T}\right)
$$

and a horizontal side corresponding to the p-type doping density in the quasineutral region. The shape of the curves emphasizes the importance of the $R_{W}$ value in the UL and LR corner of the $[n, p]$-space. $R_{W}^{[0,0]}$ is only of importance for (sufficiently large) backward bias voltages.

The paths corresponding with the simulation of the multivalent defect coincide almost entirely with the paths corresponding with the equivalent set of SRH-like defects, only at reverse bias voltages there is some small deviation. This confirms the validity of the assumption that the free carrier concentrations are similar for the simulation of the multivalent and the equivalent SRH-like set of defects.

\section{High defect density}

The simulations have been repeated with increased defect density $\left(N_{t}=N_{A}\right)$. In this case the assumption that the free carrier densities are the same in the multivalent and equivalent SRH case no longer holds, especially in the quasineutral part of the absorber the hole density deviates. However, even though the presented theory is strictly not applicable, the relation between the currents calculated in both cases also becomes significantly larger than unity for $c_{n}^{1 / 2}>10^{-8} \mathrm{~cm}^{3} / \mathrm{s}$ and the $\log \left(J_{\mathrm{SRH}} / J_{\mathrm{MUL}}\right)$ versus $c_{n}^{1 / 2}$ characteristic almost completely coincides with the characteristic calculated for a lower defect density (not illustrated here). 


\section{CONCLUSION}

The expression for the recombination rate of a multivalent defect level is very similar to the expression for a SRHlike defect and under some conditions they are equivalent. This paper investigates the conditions under which a multivalent defect can be modeled as a set of SRH-like defects, with each level having a defect density equaling the defect density of the multivalent defect as a whole.

A direct way to assess whether or not a multivalent defect can be equivalently described by a set of SRH-like defects, is the construction and interpretation of the $R_{W}$ diagram that is introduced here. Restricting to multivalent defects with three possible charge states, the $R_{W}$ parameter can easily be examined by calculating its value for the $p$-dominated, the $n$-dominated, and the deeply depleted case. In most practical cases the $p$-dominated and $n$-dominated case suffice and then one can easily state that a multivalent defect can be mimicked by an equivalent set of SRH-like defects if $c_{n}^{s+1 / 2} \ll c_{n}^{s-1 / 2}(16)$ and $c_{n}^{s+1 / 2} \gg c_{n}^{s-1 / 2}$ (17). In the case of a $p n$-junction under a large reverse bias voltage, also (19) has to be checked, which involves somewhat more elaborate calculations.

When dealing with a multivalent defect with more than three charge states, it is not possible to construct concise closed form expressions for $R_{W}$. In this more complicated case, one can study and compare the $R_{U}$-diagrams, that show the relation between the different levels belonging to the defect. Using the expressions calculated in this paper these $R_{U}$-diagrams can be visualized in a fast and easy way. Comparison of the $R_{U}$-diagrams in the multivalent case and the SRH case provides a fast way to get an indication whether or not the two different approaches lead to different behavior. If for example in the multivalent case the transition " $s-1 / 2$ " is the main recombination channel and in the SRH-like case the transition " $s+3 / 2$ " is the most important, it is obvious that the two approaches are not agreeing. The conclusion can be of help to guide further modeling.
Setting up a model for a solar cell often is a trade-off between complexity and the reliability of the simulation results. When constructing a very minimal model, the multivalent character of the defects may not be the main concern. However, when making a detailed model which should explain various measurements performed on one sample, the true character of the defect might become important. In this case, the procedure presented in the paper can be used to check in a fast way whether or not the multivalent/SRH-like character of the defects included will have an influence on the simulation results. Moreover, it can indicate whether or not the modeling can confirm/refute the assumption of having a multivalent defect.

The theory has been confirmed by simulations on a thin film solar cell structure. Where the worst equivalence between the multivalent model and its corresponding set of SRH-like defects lead to an overestimation of the current by a factor 10 , and of the recombination rate at a certain point by a factor $10^{4}$.

\section{ACKNOWLEDGMENTS}

We acknowledge the support of the Research Foundation-Flanders (K.D., FWO Ph.D. fellowship).

${ }^{1}$ C.-T. Sah and W. Shockley, Phys. Rev. 109, 1103 (1958).

${ }^{2}$ S. Siebentritt and U. Rau, Wide-Gap Chalcopyrites (Springer-Verlag, Berlin, 2006).

${ }^{3}$ S. B. Zhang, S. H. Wei, A. Zunger, and H. Katayama-Yoshiba, Phys. Rev. B 57, 9642 (1998).

${ }^{4}$ A. Milnes, Deep Impurities in Semiconductors (Wiley, New York, 1973).

${ }^{5}$ R. E. I. Schropp and M. Zeman, Amorphous and Microcrystalline Silicon Solar Cells (Kluwer Academic, Norwell, 1998).

${ }^{6}$ J. Hubin, A. V. Shah, and E. Sauvain, Philos. Mag. Lett. 66, 115 (1992).

${ }^{7}$ M. Burgelman, P. Nollet, and S. Degrave, Thin Solid Films 361-362, 527 (2007).

${ }^{8}$ K. Decock, S. Khelifi, and M. Burgelman, "Modelling multivalent defects in thin film solar cells," Thin Solid Films (to be published).

${ }^{9}$ W. Shockley and J. T. Last, Phys. Rev. 107, 392 (1957).

${ }^{10}$ Proceedings of NUMOS, Gent, 28-30 March 2007, edited by M. Burgelman, J. Verschraegen, B. Minnaert, and J. Marlein (Academia, Belgium, 2007), p. 357. 